\title{
Adoecimento por hipertensão arterial e Diabetes Mellitus: concepções de um grupo de pacientes hospitalizados
}

\author{
Conceptions of illness from hypertension and Diabetes Mellitus among a group of \\ hospital inpatients \\ Adolecer por hipertensión arterial y Diabetes Mellitus: conceptos de un grupo de \\ pacientes hospitalizados
}

\author{
Natália Pimentel Gomes Souza ${ }^{\text {I }}$ Glória Yanne Martins de Oliveira ${ }^{\text {Ill; }}$ Ana Livia Araújo Girão ${ }^{\text {III }}$; \\ Livia Marques Souzalv; Samia Jardelle Costa de Freitas Maniva ${ }^{V}$; Consuelo Helena Aires de Freitas ${ }^{\mathrm{VI}}$
}

\begin{abstract}
RESUMO: Objetivou-se identificar as necessidades de aprendizado em saúde de pacientes hipertensos e diabéticos hospitalizados. Estudo descritivo, realizado com 10 pacientes internados em um hospital público da cidade de Fortaleza-CE, nos meses de setembro a dezembro de 2011, por meio de entrevista semiestruturada e observação de campo. Os pacientes possuíam elevado grau de dependência e incapacidades graves, além de déficit de conhecimento relacionado às patologias, levando à falta de adesão ao tratamento. $\mathrm{O}$ acidente vascular cerebral foi a principal complicação clínica. A terapêutica não medicamentosa foi citada como importante, contudo a medicamentosa melhor simboliza o autocuidado para os pacientes. Concluiu-se que o enfermeiro deve implementar educação em saúde no plano de cuidados a essa clientela.

Palavras-Chave: Hipertensão; Diabetes Mellitus; educação em saúde; enfermagem.
\end{abstract}

\begin{abstract}
This descriptive study to identify health education needs among diabetic and hypertensive hospital inpatients was conducted from September to December 2011 through semi-structured interviews and field observation of 10 inpatients at a public hospital in Fortaleza. The patients were highly dependent and severely disabled, besides lacking knowledge about their pathologies, leading to failure to adhere to treatment. Strokes were the main clinical complication. Non-drug therapy was cited as important, although drug treatment symbolizes the patients' self-care better. It was concluded that nurses should perform health education in the care plan for this clientele.
\end{abstract}

Keywords: Hypertension; Diabetes Mellitus; health education; nursing.

RESUMEN: El objetivo fue identificar las necesidades de aprendizaje en salud de los pacientes diabéticos e hipertensos hospitalizados. Estudio descriptivo, realizado con 10 pacientes internados en hospital publico de Fortaleza - CE - Brasil, en los meses de septiembre a diciembre de 2011, por medio de entrevistas semiestructuradas y observación de campo. Los pacientes mostraron alto grado de dependencia y discapacidad severa, y déficit de conocimiento relacionado a las enfermedades, Ilevando a la falta de adherencia al tratamiento. El accidente cerebrovascular fue la complicación clínica principal. La terapia no farmacológica es importante, sin embargo, el autocuidado para los pacientes es el mejor fármaco. Se concluye que los enfermeros deben poner en su plan de atención educación en salud para esa clientela.

Palabras Clave: Hipertensión; Diabetes Mellitus; educación en salud; enfermería.

\section{INTRODUÇÃo}

As doenças crônicas não transmissíveis (DCNT) ganham espaço crescente no cenário de saúde. $\mathrm{O}$ destaque do presente estudo é a hipertensão arterial sistêmica (HAS) e o Diabetes Mellitus tipo 2 (DM2), considerados fatores de risco para outros agravos que acarretam elevado custo, tanto do ponto de vista econômico como social. Em virtude disso, ressalta-se a importância de ações associadas com a prevenção, diagnóstico precoce e tratamento para o controle eficaz ${ }^{1}$.

Nesse contexto, com base nas atividades desenvolvidas no Grupo de Pesquisa Saúde do Adulto e Família, da Universidade Estadual do Ceará, junto

IEnfermeira. Mestre em Cuidados Clínicos em Enfermagem e Saúde pela Universidade Estadual do Ceará. Membro do Grupo de Pesquisa Saúde do Adulto e Família. Fortaleza, Ceará, Brasil. E-mail: nataliapimentel88@yahoo.com.br.

IIMestranda do Programa de Pós-Graduação em Cuidados Clínicos em Enfermagem e Saúde da Universidade Estadual do Ceará. Membro do Grupo de Pesquisa Saúde do Adulto e Família. Fortaleza, Ceará, Brasil. E-mail: gloria yanne@hotmail.com.

IIIMestranda do Programa de Pós-Graduação em Cuidados Clínicos em Enfermagem e Saúde da Universidade Estadual do Ceará. Membro do Grupo de Pesquisa Saúde do Adulto e Família. Fortaleza, Ceará, Brasil. E-mail: liviaag_@ hotmail.com.

IVEnfermeira. Membro do Grupo de Pesquisa Saúde do Adulto e Família. Fortaleza, Ceará, Brasil. E-mail: liviaamarques@hotmail.com.

vEnfermeira. Mestre em Cuidados Clínicos e Saúde pela Universidade Estadual do Ceará. Membro do Grupo de Pesquisa Saúde do Adulto e Família. Fortaleza, Ceará, Brasil. E-mail: samia_jardelle@yahoo.com.br.

${ }^{\mathrm{V}}$ Doutora em Enfermagem. Professora do Departamento de Enfermagem. Universidade Estadual do Ceará. Líder do Grupo de Pesquisa Saúde do Adulto e Família. Fortaleza, Ceará, Brasil. E-mail: consueloaires@yahoo.com.br. 
a um hospital público terciário, percebeu-se que o adoecimento por DM e/ou HAS é um campo propício para a implementação de ações educativas pela enfermagem. Tais experiências possibilitaram a formulação de questionamentos que motivaram a realização do estudo: $\mathrm{O}$ que sabem os pacientes hospitalizados sobre DM2 e/ou HAS? Como se dá o autocuidado?

Trata-se de um recorte do projeto de pesquisa O processo saúde - doença no cotidiano de pessoas com hipertensão arterial e Diabetes Mellitus: significados e crenças na promoção do cuidado clínico de enfermagem. O objetivo do presente estudo foi identificar as necessidades de aprendizado em saúde de pacientes hipertensos e diabéticos hospitalizados relacionados à HAS e/ou DM2.

\section{REVISÃO DE LITERATURA}

Atualmente, mais de $60 \%$ dos óbitos mundiais são decorrentes de DCNT. No Brasil, tais doenças assumem papel relevante entre as principais causas de morbimortalidade ${ }^{2}$. Segundo estudos epidemiológicos, a HAS apresenta prevalência elevada e sua frequência pode variar entre $22,3 \%$ e $43,9 \%{ }^{3}$. Com relação ao DM2, este se manifesta em quase $8 \%$ da população adulta, e há uma tendência crescente ao surgimento desta doença conforme aumenta a idade, chegando a $17 \%$ nos idosos 4 .

Sabe-se que a adesão terapêutica dos pacientes com HAS e/ou DM2 significa um desafio para profissionais de saúde, entre eles, o enfermeiro, sobretudo porque o adoecimento requer cuidados diários e contínuos.

Nesse intuito, iniciativas de promoção da saúde são meios para a prevenção de agravos e controle do adoecimento ${ }^{5}$. Pois, quanto melhor o conhecimento do paciente sobre a doença, melhores serão suas ações para prevenção, adoção de hábitos saudáveis e continuidade do tratamento; ao mesmo tempo, menores serão os índices de complicações ${ }^{6,7}$.

Contudo, a adesão terapêutica ao tratamento na situação de adoecimento crônico não perpassa somente pela motivação pessoal do paciente. Diferentes nuances interferem nesse processo, tais como a baixa condição socioeconômica, a deficiência de autocuidado e de conhecimentos sobre a enfermidade, entre outros.

\section{Metodologia}

Trata-se de estudo descritivo com abordagem quantiqualitativa $^{8}$, realizado em um hospital público terciário situado na cidade de Fortaleza-CE, no período de setembro a dezembro de 2011. Participaram 10 pacientes hospitalizados, no período correspondente à coleta de dados, por complicações decorrentes de HAS e/ou DM2, selecionados por amostragem intencional9. Quanto ao critério de exclusão adotou-se: apresentar estado físico ou mental comprometido que inviabilizasse a entrevista. A delimitação dos participantes foi definida por saturação, ou seja, quando os dados, a partir da inclusão de novos participantes, passam a apresentar, na avaliação do pesquisador, certa redundância ou repetição? Para assegurar o anonimato dos participantes, atribuiu-se a cada um deles a letra E referente a entrevistado e número subsequente.

Inicialmente, houve a entrada no campo para conhecimento da dinâmica de trabalho nas unidades de internação. Foram utilizados os seguintes instrumentos de coleta: check-list, entrevista semiestruturada e observação de campo. O check-list foi adotado para identificar os pacientes diabéticos e hipertensos, mediante análise dos prontuários; o mesmo continha dados de identificação do paciente, diagnóstico e data da admissão, sexo, idade, pressão arterial e glicemia capilar. As entrevistas foram gravadas e armazenadas em arquivo de áudio, sendo a duração média de cada uma 30 minutos. As mesmas constituíram-se de duas partes, a primeira indagava sobre os dados sociodemográficos; já a segunda, continha questões relacionadas ao conhecimento sobre HAS e/ou DM2. No tocante à observação realizadas, registraram-se os dados em diário de campo. Nos pacientes com mobilidade física prejudicada, aplicou-se o Índice de Barthel (IB), avaliador do grau de dependência para as atividades de vida diária.

Os dados foram tratados pela técnica de análise categorial temática, conforme os passos recomendados a seguir ${ }^{10}$. Efetuou-se a transcrição das entrevistas na íntegra. A seguir, ocorreram leituras sucessivas, com vistas à exploração do material obtido e recorte do texto em unidades de registro. Formularam-se as seguintes categorias temáticas: Conhecimentos dos pacientes sobre a doença (HAS e/ou DM2) e Principais cuidados com a saúde. A análise dos conteúdos respaldou-se na literatura pertinente ao tema.

$\mathrm{Na}$ abordagem quantitativa, foram calculadas a frequência absoluta e a média aritmética, conforme a análise estatística.

Cumpriram-se os preceitos éticos e legais em pesquisa com seres humanos ${ }^{11}$. Como recomendado, a coleta dos dados ocorreu mediante a assinatura do Termo de Consentimento Livre e Esclarecido dos participantes. O projeto de pesquisa foi aprovado pelo Comitê de Ética em Pesquisa da Universidade Estadual do Ceará, sob o parecer de no 05050534-3.

\section{Resultados e Discussão}

\section{Caracterização dos participantes do estudo}

Investigaram-se 10 pacientes hospitalizados, dois eram portadores concomitantemente de DM e HAS, quatro de HAS e quatro de DM, isoladamente. A idade média foi de 59,6 anos. Dos pesquisados, sete eram homens e três eram mulheres. Foram identifi- 
cados estados civis diversificados: homens e mulheres casados ou vivendo em união consensual, solteiros, viúvos e divorciados. Ressalta-se cinco pacientes residiam com o cônjuge feminino e três com as filhas, as quais assumiriam o cuidado dos pacientes após a alta hospitalar.

Encontrou-se baixo nível de escolaridade, com destaque para o ensino fundamental incompleto. A escolaridade média foi de 4,7 anos de estudo. A renda familiar médio foi de R\$ 934,2/mês. Quanto à ocupação, cinco eram aposentados, dois desenvolviam atividades informais, dois possuíam trabalho formal e um estava desempregado.

Considerando a renda reduzida e a baixa escolaridade dos sujeitos pesquisados, o nível socioeconômico mais baixo está associado à maior prevalência de hipertensão arterial, além de maior risco de lesão em órgãos alvo e eventos cardiovasculares, como resultado do menor acesso aos cuidados de saúde ${ }^{12}$.

Com relação à identificação de outros fatores de risco modificáveis, três pessoas eram fumantes, duas faziam uso regularmente de álcool, oito eram sedentárias. Apenas duas praticavam atividade física na modalidade de caminhada, três vezes por semana. Os valores da pressão arterial variaram entre 140 179/80-110. Entre os pacientes diabéticos, a glicemia capilar média foi de $197 \mathrm{mg} / \mathrm{dL}$.

Segundo constatado, a principal complicação clínica foi o acidente vascular encefálico (AVE), diagnosticado em cinco pessoas. Três pessoas foram hospitalizadas por complicações diabéticas, entre elas o pé diabético, manifestado em dois pacientes.

O AVE é uma das principais causas de óbito na população adulta, e tem como principais fatores de risco a HAS e o DM2. No entanto, a mortalidade é apenas uma das medidas de saúde pública do impacto do AVE. Outra igualmente importante é a incapacidade física ${ }^{13}$.

A incidência do AVE é maior após os 65 anos, havendo um aumento do risco com a idade. $\mathrm{O}$ envelhecimento da população, associado a fatores de risco para doença cerebrovascular ${ }^{14}$ como HAS, DM2, tabagismo, consumo de álcool, dislipidemia e obesidade, explicam, em parte, a alta ocorrência de AVE.

Desse modo, buscou-se conhecer o estado de saúde destes pacientes no tocante ao grau de dependência para as atividades de vida diária, utilizando o IB. Assim, segundo verificado, três pacientes possuíam dependência moderada, com pontuação variada entre 65 e 80; um revelava dependência severa, pontuando 50; e um dependência leve, com IB equivalente a 90 pontos. Os pacientes com dependência severa e moderada denotaram maior propensão ao desenvolvimento de úlceras por pressão. Três deles tinham lesões de pele em pelo menos uma das regiões corporais: sacral, troncantérica e calcâneos.
O IB é uma escala que orienta o cuidado de pacientes em progresso da capacidade funcional. Nesse âmbito, uma pontuação inicial superior a 60 relaciona-se com menor duração do período de hospitalização e maior probabilidade de reintegrar-se à vida na comunidade após a alta hospitalar ${ }^{15}$.

Após a alta hospitalar, cerca de $80 \%$ dos pacientes com AVE continuam necessitados de cuidados especiais em virtude das incapacidades residuais ${ }^{16}$. Dessa forma, na maioria dos casos, a família, na figura do cuidador informal ou familiar, assume a responsabilidade dos cuidados. No entanto, para assegurar a continuidade no tratamento e a prevenção de complicações é preciso preparar paciente e cuidador familiar, por meio da educação em saúde.

Mostrou-se relevante caracterizar os pacientes hospitalizados por predizer os cuidados a serem planejados e executados pelos profissionais de saúde, entre eles o enfermeiro.

\section{Conhecimento dos pacientes sobre a doença}

Para desempenhar o autocuidado, a pessoa hipertensa e/ou diabética precisa de conhecimentos e habilidades específicos para o alcance dos resultados. Assim, os pacientes foram questionados sobre o adoecimento. Vários depoimentos demonstraram déficit de conhecimento. Encontrou-se a construção simbólica acerca da doença, como explicitado nas falas:

Diabetes é o sangue doce, é açúcar no sangue. (E2)

Sobre diabetes, sei que não posso comer açúcar. (E10)

Quando a pressão está alta, eu sinto dor de cabeça. (E6)

Segundo o referencial teórico do interacionismo simbólico, as proposições expostas ocorrem como resultado de uma interação das pessoas dentro de uma estrutura social, de suas ações humanas como participantes do mundo ${ }^{12}$. Assim, as declarações obtidas são decorrentes das experiências de vida individuais e comunitárias, tendo as doenças, para cada entrevistado, significados únicos oriundos de suas vivências como sujeitos no mundo.

Por serem patologias crônicas, o tratamento da HAS e DM2 é permanente. Nesse sentido, para adesão ao plano terapêutico, faz-se necessário o esclarecimento sistemático visando orientar o paciente acerca da doença e suas consequências em longo prazo, bem como dos benefícios advindos do alcance e da manutenção das metas instituídas ${ }^{13,17}$.

A situação encontrada no estudo mostrou-se preocupante, pois essas pessoas realizavam tratamento para HAS e/ou DM2 há um período de tempo superior a 5 anos, e ainda demonstravam desconhecimento sobre a doença, interferindo no autocuidado. Assim, alguns pacientes negligenciaram o cuidado com a saúde, demonstrando a falta de adesão ao tratamento, como se percebe nos discursos: 


\section{Eu não faço dieta, como o que quero. (E4)}

Eu como comida gordurosa, comida salgada, nem os remédios da pressão eu tomo direito. (E6)

Nesse contexto, as orientações educativas, além de informar sobre a doença, auxiliam o paciente no reconhecimento de sintomas de descontrole da HAS e/ou DM2, bem como sobre os efeitos colaterais e secundários de eventuais medicamentos em uso. Portanto, as intervenções educativas objetivam prevenir sequelas, internações e óbitos relacionados com complicações agudas e crônicas dessas doenças. Como principal estratégia, busca-se garantir a adesão ao tratamento prescrito e a adoção de hábitos de vida saudável ${ }^{13,17}$.

Outros pacientes definiram HAS e/ou DM2 com base nas complicações clínicas motivadoras da hospitalização, o AVE, a retinopatia e o pé diabético. As afirmativas decorriam da experiência de adoecimento.

Hipertensão é o perigo de trombose. (E1)

Diabetes afeta a visão e causa feridas. (E5)

Diante dos depoimentos, o adoecimento ainda se caracteriza como um mundo desconhecido, pois os pacientes detinham um saber mínimo. $O$ conhecimento da condição de saúde e dos meios para melhorá-la é fundamental para a manutenção da qualidade de vida. Portanto, cabe ao enfermeiro proporcionar informações que os sensibilizem para o devido autocuidado.

No tratamento de hipertensos e/ou diabéticos, a pedra angular reside no controle dos níveis pressóricos e glicêmicos. Não obstante, o cuidado a essa clientela deve englobar estratégias de promoção de qualidade de vida e bem-estar ${ }^{18}$. Nesse contexto, a educação em saúde se apresenta como estratégia fundamental do cuidado, sendo ela um instrumento facilitador para a capacitação da comunidade, contribuindo para a promoção da saúde. Assim, profissionais de saúde e usuários necessitam estabelecer uma relação dialógica pautada na escuta terapêutica, no respeito e na valorização das experiências, das histórias de vida e da visão de mundo ${ }^{19}$.

Observou-se no cenário pesquisado e foi registrado no diário de campo que muitos não promoviam nenhuma atividade educativa relacionada à HAS e/ou DM2 ou aos cuidados domiciliares. Outros, quando a realizavam, limitavam-se à disseminação de informações isoladas durante a execução de algum procedimento, sem o planejamento adequado às carências educativas dos pacientes. Segundo evidenciado, percebeu-se a fragilidade na prática de educação em saúde dos profissionais no local da pesquisa.

Caberia aos profissionais de saúde, em especial, ao enfermeiro, a implementação de um plano de cuidados individualizado, com esclarecimento de dúvidas, repasse de orientações, oportunizando aos pacientes verbalizar suas experiências de vida, e o modo como cuidam da própria saúde.
Nas falas ocorreram questionamentos relacionados às doenças, reforçando o déficit de conhecimento.

Posso ficar curado tomando apenas os remédios? (E1)

Posso comer açúcar de alguma maneira? Tenho dificuldade em usar o adoçante. (E4)

Gostaria de aprender sobre minha doença [diabetes]. (E7)

Como se depreende, os pacientes desejavam conhecer mais sobre o processo de adoecimento, revelando que o período de hospitalização também é propício para o desenvolvimento de intervenções educativas. Logo, é essencial proporcionar o conhecimento necessário para fortalecer o autocuidado, e permitir que ações terapêuticas sejam construídas conjuntamente com os pacientes e incorporadas à vida de cada um deles.

O papel fundamental do homem é o de ser sujeito e não objeto de transformação, tarefa que dele exige, durante sua ação sobre a realidade, um aprofundamento da sua tomada de consciência do real, objeto de atos contraditórios daqueles que pretendem mantê-la como está e dos que pretendem transformála. Portanto, se a vocação ontológica do homem é a de ser sujeito e não objeto, só poderá desenvolvê-la na medida em que, refletindo sobre suas condições, introduz-se nelas, de modo crítico e criativo, não agindo como mero espectador, mas intervindo nela ${ }^{20}$.

Através do processo educativo, são descobertas possibilidades de adaptação à doença crônica, levando em consideração o estilo de vida individual, com seu contexto familiar e suas dimensões de trabalho e de socialização, além da criação de agentes promotores de saúde, proporcionando apoio e respeito mútuo entre usuários e equipe que deve atuar como cooperadora dessa promoção.

\section{Principais cuidados com a saúde}

O principal cuidado relatado foi o uso diário dos medicamentos:

Tomo os remédios, não deixo faltar. (E1)

Contudo, é preciso se esclarecer que o controle da HAS e/ou DM2 não reside apenas nessa medida, outros cuidados devem ser adotados em igual importância para se atingir resultados satisfatórios.

Grande parte das pesquisas aborda a adesão à prescrição medicamentosa e reforça sua importância. Entretanto, as mudanças dos hábitos alimentares e de estilo de vida, isto é, o tratamento não medicamentoso, são preconizados a todos os pacientes, independente do estágio da doença ${ }^{17}$.

O tratamento da HAS e/ou DM perdura por toda a vida, sem ou com medicamentos. $\mathrm{O}$ primeiro é feito mediante controle do peso, melhora do padrão alimentar, redução do consumo do sal, moderação no consumo de bebidas alcoólicas, prática regular de 
exercício físico, abstenção do tabagismo e controle do estresse psicoemocional; já o medicamentoso tem por base o uso de drogas ${ }^{15}$.

Outro cuidado mencionado por duas pessoas foi a prática exercício físico anteriormente à hospitalização.

Faço caminhada três vezes por semana. (E4)

Tal prática representa uma medida benéfica para a diminuição das variáveis clínicas de pacientes com HAS e/ou DM2, sobretudo com relação aos níveis glicêmicos e índice de massa corporal, além de compor o plano terapêutico e propiciar maior convívio social ${ }^{18}$.

O seguimento de dieta adequada foi citado por um hipertenso e uma diabética, segundo se observa nas falas:

Procuro comer com pouco sal, pouca gordura. (E10)

Tem que fazer a dieta, comer sem açúcar. (E5)

Contudo, grande parte do grupo referiu dificuldade no controle dietético, principalmente referente ao consumo de sal:

\section{Tento diminuir o sal, mas é difícil. (E2)}

Eu como comida salgada. (E7)

Esses achados coincidem com os dados de outro estudo $^{18}$, no qual a principal dificuldade encontrada no tratamento da HAS foi a adoção de dieta hipossódica. Outros estudos também apresentam resultados semelhantes ${ }^{21,22}$.

Diante do exposto, percebe-se que intervenções educativas são necessárias. A educação em saúde das pessoas com HAS e DM2 tem por finalidade influenciar o comportamento do paciente na obtenção de mudanças efetivas ${ }^{3}$, instrumentalizando-o para que, por meio dos seus próprios recursos, desenvolva mecanismos para identificar e prevenir complicações.

\section{CONCLUSÃo}

O estudo possibilitou reflexões acerca do cuidado nos adoecimentos por HAS e DM. Nesse sentido, a atuação do enfermeiro junto a pacientes e familiares na implementação da educação em saúde com vistas ao autocuidado é indispensável, seja no domicílio ou nos serviços de saúde.

A partir das categorias temáticas apresentadas Conhecimentos dos pacientes sobre a doença (HAS e/ou DM) e Principais cuidados com a saúde-, evidenciaramse a presença de complicações decorrentes dos adoecimentos de base e o desconhecimento dos participantes com relação à prática das atividades de autocuidado.

Os resultados do estudo não podem, no entanto, ser generalizados, pois se reconhece suas limitações, sobretudo no tocante ao reduzido número de participantes e único cenário. Todavia, espera-se fornecer subsídios para a prática profissional do enfermeiro que atua junto a essa clientela. Da mesma forma, pretendese explicitar o caminho percorrido para que outros pesquisadores possam confirmar ou refutar os achados.

\section{REFERÊNCIAS}

1.Ribeiro AG, Cotta RMM, Ribeiro SMR. A promoção da saúde e a prevenção integrada dos fatores de risco para doenças cardiovasculares. Ciênc saúde coletiva. 2012; 17:7-17.

2.Casado L, Vianna LM, Thuler LCS. Fatores de risco para doenças crônicas não transmissíveis no Brasil: uma revisão sistemática. Rev Bras Cancerol. 2009; 55: 379-88. 3.Pierin AMG, Jesus ES, Augusto MAO, Gusmão J, Ortega K, Mion Júnior D. Variáveis biopsicossociais e atitudes frente ao tratamento influenciam a hipertensão complicada. Arq Bras Cardiol. 2010; 95:648-54.

4.American Diabetes Association. Diagnosis and classification of diabetes mellitus. Diabetes Care. 2008; 31 (1 supl):55-60.

5.Torres HC, Souza ER, Lima MHM, Bodstein RC. Intervenção educativa para o autocuidado de indivíduos com diabetes mellitus. Acta Paul Enferm. 2011; 24:514-9. 6.Olinda QB, Silva CAB. Retrospectiva do discurso sobre promoção da saúde e as políticas sociais. Rev Bras Promoç Saúde. 2007; 20(2): 65-7.

7.Felipe GF, Silveira LC, Moreira TMM, Freitas MC. Presença implicada e em reserva do enfermeiro na educação em saúde à pessoa com hipertensão. Rev enferm UERJ. 2012; 20:45-9.

8.Polit D, Hungler B. Fundamentos de pesquisa em enfermagem: avaliação de evidências para a prática de enfermagem. $7^{\text {a }}$ ed. Porto Alegre (RS): Artmed; 2011.

9.Fontanella BJB, Ricas J, Turato ER. Amostragem por saturação teórica em pesquisas qualitativas em saúde: contribuições teóricas. Cad Saúde Pública. 2008; 24:17-27. 10.Bardin L. Análise de conteúdo. Lisboa (Pt): Edições 70 Persona; 2010.

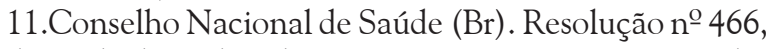
de 12 de dezembro de 2012. Diretrizes e normas regulamentadoras para pesquisas envolvendo seres humanos. Brasília (DF): CNS; 2012.

12.Charon JM. Symbolic interacionism. $3^{\text {a }}$ ed. Englewood Cliffs (USA): Prentice-Hall; 1989

13.Sociedade Brasileira de Cardiologia. Diretrizes Brasileiras de Hipertensão Arterial. V. In: Consenso Brasileiro de Hipertensão Arterial. Rio de Janeiro: SBC; 2010. 14.Giles MF, Rothwell PM. Measuring the prevalence of stroke. Neuroepidemiology. 2008; 30:205-6.

15.Cavalcante TF, Moreira RP, Araujo TL, Lopes MVO. Demographic factors and risk indicators of stroke:comparison between inhabitants of Fortaleza Municipal district and the national profile. Rev LatinoAm Enfermagem. 2010; 18: 703-8.

16.Cardoso JH, Costa JSD. Características epidemiológicas, capacidade funcional, fatores associados em idosos de um plano de saúde. Ciênc saúde coletiva. 2010; 15: 2871-8. 17.Sociedade Brasileira de Diabetes. Consenso brasileiro sobre diabetes - diagnóstico e classificação do Diabetes 
Meliltus e tratamento do Diabetes Meliltus tipo 2. 2009. [citado em 15 dez 2014]. Disponível em: http://www. sbd.org.br

18. Figueiredo NN, Asakura L. Adesão ao tratamento anti-hipertensivo: dificuldades relatadas por indivíduos hipertensos. Acta Paul Enferm. 2010; 23:782-7.

19.Cervera DPP, Parreira BBD, Goulart BF. Educação em saúde: percepção dos enfermeiros da atenção básica em Uberaba (MG). Ciênc saúde coletiva [online]. 2011; 1547-54.
20.Freire P. Educação e mudança. 27ª ed. São Paulo. Ed. Paz e Terra; 2003.

21.Silva LMC, Palha FP, Barbosa GR, Protti ST, Ramos AS. Aposentados com diabetes tipo 2 na saúde da família em Ribeirão Preto, São Paulo - Brasil. Rev esc enferm USP. 2010; 44:462-8.

22.Dosse C, Cesarino CB, Martin JFV, Castedo MCA. Fatores associados à não adesão dos pacientes ao tratamento de hipertensão arterial. Rev Latino-Am Enfermagem. 2009:17: 201-6. 